\title{
Impact of Optical Hop Constraints on the IP/MPLS-over-WSON Networks CAPEX
}

\author{
Fengqing Liu ${ }^{1}$, Jaume Comellas ${ }^{2}$ \\ ${ }^{1}$ School of Opto-Electronic Engineering, Nanjing University of Posts and Telecommunications, Nanjing, China \\ 2 Dept. of Signal Theory and Communications, Universitat Politècnica de Catalunya Barcelona, Spain
}

\begin{abstract}
In this work, the impact of hop constraints on the CAPEX of multi-layer IP/MPLS over WSON networks is evaluated under different scenarios. By routing lightpaths with different number of transparent hops, the cost of networks varies significantly. This effect is thoroughly analyzed with a heuristic algorithm for different network topologies belonging to some European network operators. The main conclusion extracted is that limiting the transparent reach in such multi-layer networks can give a good tradeoff between CAPEX of IP/MPLS networks and WSON networks, and even lead to significant savings in CAPEX when compared to longer lightpaths routing strategies. Key words: IP/MPLS over WSON networks; Optical Hop constraints; CAPEX; heuristics
\end{abstract}

\section{Introduction}

IP over WDM network design and analysis has received significant research attention over the past years ${ }^{[1]}$. The appropriate collaboration between both layers has been a key issue in network research for more than 10 years, and has not yet been completely solved as many new constraints have been introduced as technology progresses. Some of these constraints have been thoroughly studied in the literature, and have generated lots of results and controversial. Multi-layer networks (MLN) integrating both packet (IP Multi-Protocol Label Switching, IP/MPLS) as well as optical (Wavelength Switched Optical Networks, WSON) switching, are seen anyway as a $\operatorname{cost}^{[2-3]}$ and energy ${ }^{[4]}$ efficient solution for deploying transport networks, since they combine the bandwidth flexibility and the coarse transport capacity, provided respectively by IP/MPLS and WSON.
While optical stuff is recognized as the most appropriate solution for huge bit-rate connections transport, it lacks from suitable granularity to cope with the smaller client demands capacity. Many works have been devoted to the optical grooming issue since the end of 1990s ${ }^{[5-6]}$. Therefore, one important research topic is related to the mismatching between granularities in the optical and electrical domains. How to group client connection demands to efficiently fill these optical channels has been one of the most important areas of research related to optical networks. The multi-layer traffic engineering concept ${ }^{[7]}$ emerged as a very important topic when going to GMPLS controlled networks, because coordination among the higher and lower layers is essential in improving the whole network resources usage. Focusing on the optical layer, and trying to improve the efficiency of optical spectrum resource over networks, there has been introduced a new concept during recent years known as elastic networks ${ }^{[8]}$. The main idea behind elastic optical networks consists in using simultaneously different bit rates and different modulation formats to improve wavelengths occupancy and the whole network efficiency.

To get the optimum bandwidth efficiency while reducing the network CAPEX, the collaboration between optical bypass (provided by WSON) and traffic grooming chances (given by IP/MPLS) is crucial. In general, long lightpaths going through large number of hops make full use of optical bypass, while they make client low-granularity demands having fewer chances to be grouped into a lightpath. The opposite effect is experienced for short lightpaths. By appropriately adjusting the transparent hop 
constraints of lightpaths (routing lightpaths with the appropriate number of transparent hops), the benefits of both, optical bypass and traffic grooming, can be attained. Avoiding signal degradation by the effect of physical impairments is another reason to limit the number of hops that lightpaths can go through. As optical components in a node introduce non-linear effects, crosstalk, and some other impacts, optical signal traversing a node suffers degradation. Some previous works focus on physical impairments and restrict the maximum transparent reach of lightpaths ${ }^{[9-12]}$ to limit their effects. The main purpose of this work consists in analyzing the impact of optical bypassing and traffic grooming capabilities, provided by IP/MPLS over WSON network nodes, on the network CAPEX. Hop constraints are used as a means to provide coordination between them.

The remainder of the paper is organized as follows. In Section 2 the network and cost model considered during the study is introduced. The problem description and a novel heuristic algorithm to solve it are presented in Section 3. Then, Section 4 provides some numerical results obtained considering different network topologies and traffic demand profiles, and finally Section 5 draws up the main conclusions.

\section{Network and Cost Model}

In IP/MPLS over WSON multi-layer networks, when a new client connection demand (which is known as Label Switched Path, LSP, in the MPLS world) arrives, one or more lightpaths may be set up between its source and destination node. According to how optical bypass is allowed, the following lightpath establishment strategies can be applied:

1) Single lightpath. One single source to destination lightpath is set up to accommodate the client LSP. Therefore, intermediate nodes are optically bypassed, and no grooming capabilities can be then used at these intermediate nodes.

2) Link-by-link. A different lightpath is created between each pair of adjacent nodes from source to destination. As traffic is converted from optical to electrical at each intermediate node, the grooming chances are maximized and other LSPs using the same link can be grouped over that lightpath if there is available bandwidth.

3) With hop constraints. In this case, the created lightpaths have a maximum number of transparent hops $r$. Then, more than one lightpath is necessary if hops number of the path is greater than $r$. The advantages as well as the drawbacks of the previous strategies are then smoothed and this scheme can be seen as a trade-off solution. Depending on the instantaneous requirements, the network can sacrifice transparency to have a better usage of the established lightpaths bandwidth.

The network nodes architecture assumed in this work is depicted in figure 1. Each node is composed by an IP/MPLS router attached to an all-optical cross-connect (OXC). An IP/MPLS router is subdivided into two building blocks: the basic node (includes router chassis, switch matrix, etc), which can be classified as different router classes according to its ports number and switching capacity, and the opto-electronic (OE) ports. An OXC node has three blocks: WSS, optical amplifiers (pre-amplifier and post-amplifier), and splitters. When routing a demand, the best option to assign the network resources must be decided to improve the resource utilization. While sometimes it is worth establishing a new lightpath for the current routing demands, re-using an already existing lightpath can be more efficient in some other cases, always taking into account the whole network performance. Considering the cost of all the elements included in the network (OE ports, IP/MPLS processing, WSS, in-line optical amplifiers, fibre links...), it is possible to find the minimum cost under hop constraints given an actual offered traffic profile. This is the main objective of this work and will be evaluated in 


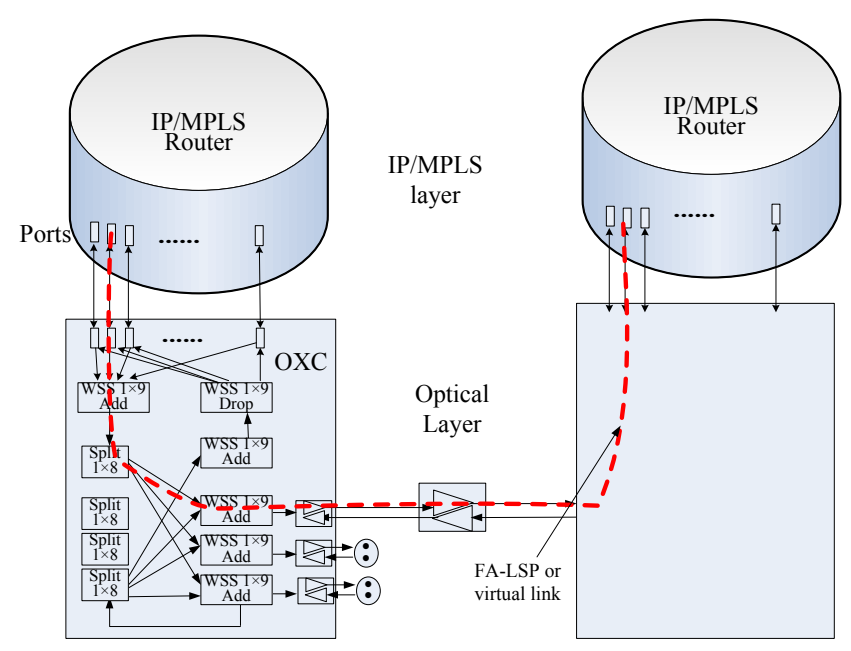

Figure 1 Node architecture of the IP/MPLS over WSON network considered.

next Sections. Obviously, the maximum allowed transparent hops of the lightpaths (hereafter referred as hops constraint) has a great impact on the cost of the network.

The cost computation is based on figure 1 components cost. Total network cost is calculated as the sum of the cost of IP/MPLS topology $\left(\operatorname{cost}_{I P}\right)$ and optical topology $\left(\operatorname{cost}_{O T}\right)$. cost $_{I P}$ is the sum of the cost of IP/MPLS router class (cost $\left.t_{\text {rclass}}\right)$ and $\mathrm{OE}$ ports (cost $t_{\text {port }}$ ), while cost $_{O T}$ corresponds to the sum of the cost of OXC ( $\left.\operatorname{cost}_{o x c}\right)$, optical amplifiers $\left(\operatorname{cost}_{O A S}\right)$ and fiber links ( cost $\left._{f i b e r}\right)$. That is

$$
\begin{gathered}
\cos t_{I P}=\cos t_{\text {rclass }}+\cos t_{\text {port }} \\
\cos t_{O T}=\cos t_{O X C}+\cos t_{O A S}+\cos t_{\text {fiber }}
\end{gathered}
$$

The different components cost is calculated as follows:

$$
\begin{aligned}
& \cos t_{\text {rclass }}= \sum_{v \in V, i \in R} r c_{i} \times \gamma_{i}^{v} \\
& \cos t_{p o r t}=2 \times \sum_{e \in E, j \in P} p c_{j} \times \rho_{j}^{e} \\
& \cos t_{O X C} \cong \sum_{n \in N}\left[3 \times \cos t_{W S S}+\right. \\
&\left.N_{n} \times\left(\cos t_{W S S}+\text { Cost }_{O A}\right)\right] \\
& \cos t_{O A S}=\sum_{l \in L} \zeta_{l} \times\left(\left|\frac{\text { len }}{\text { AS }}\right|-1\right) \times \cos t_{O A} \\
& \cos t_{\text {fiber }}=\sum_{e \in E, l \in L} \lambda_{l}^{e} \times \text { len }_{l} \times \cos t_{F C}
\end{aligned}
$$

In equation (3), $r C_{i}$ corresponds to the cost of router class $i, \gamma_{i}^{v}=1$ if node $v$ is configured as router class $i$. In equation (4), $p c_{j}$ is the cost of port class $j$, and $\rho_{j}^{e}=1$ if lightpath $e$ uses port class $j$. As the cost of splitters is negligible, the cost of OXC mainly includes the cost of WSS and optical amplifiers. An OXC node can have multiple WDM links (input/output fibers), and each one has its own WSS and optical amplifier. In addition, three other WSS are needed to add or drop demands. So, the total cost of OXC node is calculated as equation (5), in which $N_{n}$ is the number of WDM links configured in node $n$. The cost of in-line amplifiers are decided by the link length $L(\mathrm{~km})$ and amplifier span length $(A S(\mathrm{~km}))$. Its total cost can be calculated using equation (6), where $\zeta_{l}$ is the number of fibers used in link $l$. In equation (7), $\lambda_{l}^{e}$ is set to 1 if lightpath $e$ goes through link $l$, and $\cos t_{F C}$ is the cost per $\mathrm{km}$ per channel of using the optical fibers.

\section{Problem description and algorithms for IP/MPLS-over-WSON networks with hop constraints}

The main objective of this section consists in finding the minimum network cost as a function of the hop constraints. To obtain the optimum cost value of multilayer IP/MPLS over WSON networks under hop constraints, the following 
problem is solved:

\section{Given:}

(1) the optical topology represented by a graph $G_{o}(N, L)$, being $N$ the set of OXC nodes and $L$ the set of bidirectional fiber links connecting two OXC nodes;

(2) the virtual network represented by a graph $G_{v}(V, E)$, being $V$ the subset of nodes $N$ where IP/MPLS nodes can be placed, and $E$ the set of virtual links defining the connectivity among the IP/MPLS nodes;

(3) hop constraints $r$, the maximum route length (hops) for a lightpath;

(4) a set $D$ of IP/MPLS demands (client LSP) to be transported;

(5) IP/MPLS equipment cost, specified by a fixed cost for every type of IP/MPLS node and $\mathrm{OE}$ port;

(6) Optical equipment cost, which includes a fixed cost for common hardware, and a variable cost which depends on the nodal degree;

(7) A cost for every optical amplifier $\left(\cos t_{O A}\right)$ to be equipped in the used fiber links and a cost per $\mathrm{km}$ per channel $\left(\cos t_{F C}\right)$ of using the deployed fiber;

\section{Output:}

(1) The optical network resources, including optical nodes with the number of WDM links and fibers, and optical amplifiers configured in each fiber;

(2) The configuration of every IP/MPLS node in terms of switching capacity, and number and bit-rate of $\mathrm{OE}$ ports;

(3) The set of used virtual links (lightpaths), including their routes over the optical network;

(4) The route of every demand over the virtual topology.

\section{Objective:}

Minimize the expected cost for the network designed for the given set of demands under hop constraints.
Due to hop constraints, more than one lightpath may have to be established to allocate a single demand. In detail, when a new demand has to be served and its length is greater than $r$, the following procedure will be used: establish as many $r$ hops lightpaths as necessary and, ensure that only the last part of the path is covered with a lightpath whose number of hops is smaller than $r$. In other words, with the exception of the last lightpath which has the same destination node as the demand, the lightpaths created are exactly $r$ hops (the objective consists in using lightpaths as long as possible, but never longer than $r$ hops), which can be expressed using the following equations:

$$
\begin{gathered}
\sum_{l \in L} \lambda_{l}^{e}-M \times\left(\chi_{e}^{p}-1\right) \geq r \\
\forall p \in P, e \in E, N d(e) \neq S D_{d}(p) \\
\sum_{l \in L} \lambda_{l}^{e}+M \times\left(\chi_{e}^{p}-1\right) \leq r \forall p \in P, e \in E
\end{gathered}
$$

In the above equations, $p$ is a demand-decided virtual path, which can be divided into one or more lightpaths. $e$ is a lightpath or a virtual link. $N d(e)$ and $S D_{d}(p)$ are the destination nodes of $e$ and $p$, respectively. $M$ is a big enough integer. $\chi_{e}^{p}$ is set to 1 if $p$ includes $e$, and is set to 0 otherwise. $\lambda_{1}^{e}$ is set to 1 if lightpath $e$ goes through fiber link $l$, otherwise 0 .

The problem can be modeled as an ILP (Integer Linear Programming) problem. However, its exact solution will be very hard to be derived with real-sized network and traffic instances. Due to this reason, we develop a novel heuristic algorithm to solve the problem. The main idea behind this heuristic consists in routing the demands (client LSPs), by selecting the route that conforms to the above hop constraints, while leading to the minimal incremental cost. When calculating the route, both existing and 


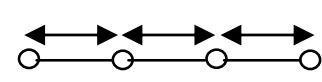

(a)

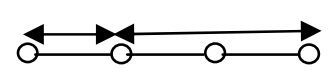

(b)

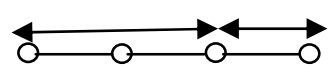

(c)

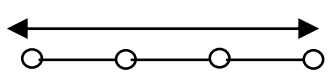

(d)

Figure 2 Different possibilities for lightpath establishment in a three hops path $(m=3)$. (a) Three one hop lightpaths, (b) One one hop plus one two hops lightpaths, (c) One two hops plus one one hop lightpath, (d) One three hops lightpath.

potential lightpaths are considered. The existing lightpath refers to an already set-up lightpath which has residual capacity bigger than the bandwidth required. The potential lightpath refers to a lightpath that may be setup taking into account the existing hop constraints. Each node pair will be assigned a cost weight based on its lightpath state, in which a weighted graph is derived. Although the incremental cost for existing lightpaths is 0 , a small positive value is set to force the client LSP to use as less existing lightpaths as possible. The cost of bringing up the potential lightpath includes the cost of its two ports, router class upgrade, added fiber channels and added OXC trunks if necessary. Dijkstra algorithm is used on the derived graph to get the minimum incremental cost route. If the derived route goes though a potential lightpath, it will be actually brought up.

$K$ shortest paths are calculated in advance for each source-destination node pair. To make the first $\mathrm{n}-1(\mathrm{n} \geq 1)$ lightpaths go through $r$ hops and the last one less than or equal to $r$ hops, a potential lightpath is only allowed between a node pair if the following conditions are satisfied:

1. Its destination node is different than that of the demand and one of $K$ paths goes through $r$ hops;

2. Its destination node is the same as that of the demand and there is one of $K$ paths that goes through less than or equal to $r$ hops.

Consider the solution space with and without hop constraints. The difference between them is the lightpath establishment strategy. Without hop constraints, all the possibilities for lightpath establishment in a given path will be considered. As an example, if the path length is $m$, there are $2^{m-1}$ different lightpaths that can potentially be established in the path without hop constraints (due to space consideration, it is not proved here). When $\mathrm{m}=2,2$ possibilities can be found, i.e. two one-hop lightpaths or one two-hop lightpath. For $\mathrm{m}=3$, there are 4 different chances (see figure 2 for better understanding). In our heuristic algorithm, and considering the case with $r=2$, the option shown in figure. 2 (c) will be chosen if possible. The solution space is therefore reduced from $2^{m-1}$ to 1 . It should be noted that there are still $K$ types of lightpath establishment that can be chosen between a source-destination node pairs, because there is one type of lightpath establishment for each of the $K$ shortest paths calculated in advance.

\section{Illustrative Numerical results}

In this section, three optical network topologies are used as examples. These three topologies (depicted in figure 3) are the 20-node British Telecom (BT), the 21-node Spanish Telefonica (TEL), and the 21-node Deutsche Telecom (DT) networks.

It has to be noted that all data derived is an average of 5-times simulation results. During simulations, two demand traffic profiles are considered: the first one corresponds to a lightly loaded network with an aggregated demand of 5Tbps; the second one (7Tbps), corresponds to a heavily loaded network. Demands (client LSPs) are uniformly distributed between every node pairs. The connection demands bit-rate values are also randomly generated according to the following ratio: 1Gbps: 10Gbps: 40Gbps: 


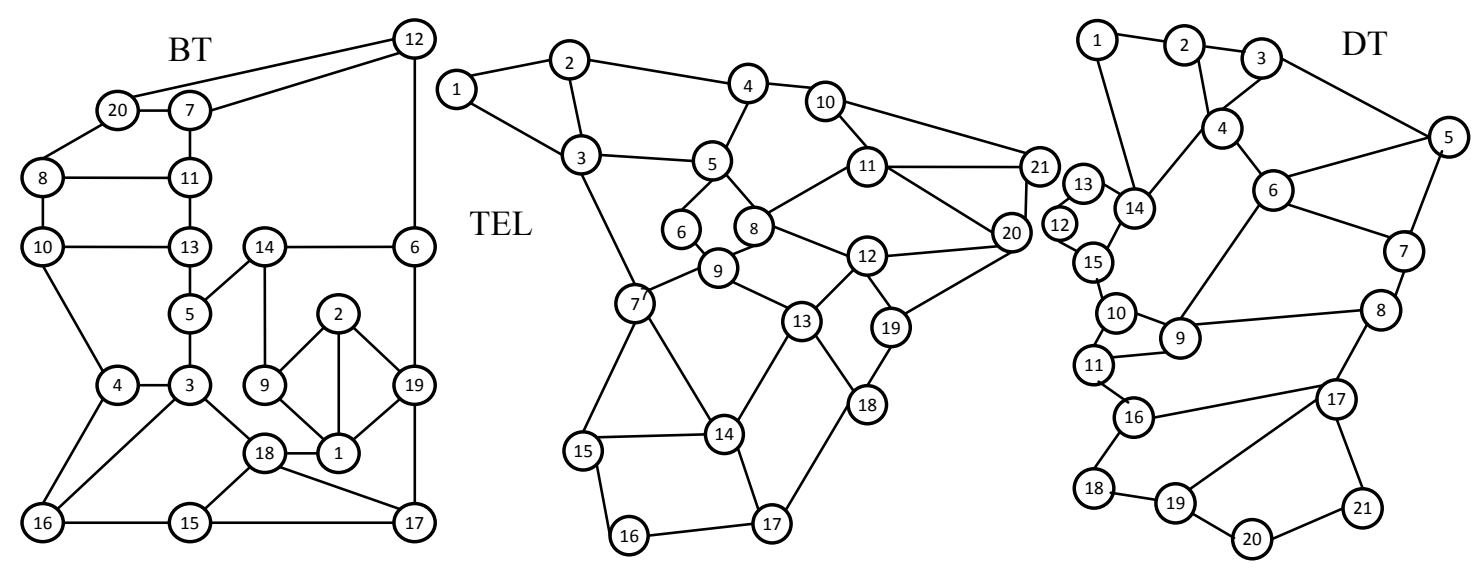

Figure 3 Optical network topologies used: BT, TEL, and DT.

Table 1 Network equipment cost values assumed for the different elements

\begin{tabular}{|c|c|c|c|c|}
\hline Ports & 1Gbps & 10Gbps & 40Gbps & 100Gbps \\
\hline Port cost (cu) & 0.5 & 1.5 & 3 & 6 \\
\hline \multicolumn{2}{|c|}{ WSS cost (cu) } & \multicolumn{2}{|c|}{10} \\
\hline \multicolumn{2}{|c|}{ OA cost (cu) } & \multicolumn{2}{|c|}{2} \\
\hline Cost per km per channel (cu) & \multicolumn{2}{|c|}{0.02} \\
\hline
\end{tabular}

$100 \mathrm{Gbps}=100: 20: 5: 2$. It is assumed that there are 80 wavelengths available in each fiber link, and lightpaths capacity is selected among $1 \mathrm{Gbps}$, $10 \mathrm{Gbps}$, 40Gbps, and 100Gbps. The value of $K$ is set to 10 during simulations, so 10 paths are calculated in advance for each source-destination node pair. The network equipment cost (given in arbitrary cost units, cu), as well as technical characteristics, are shown in Table 1. As the diameter of the topologies considered are 5, 6 and $8, r$ values range from 1 to 5,6 and 8 , respectively for BT, TEL and DT networks. Let us remind that $r$ has to be understood as the maximum number of optical links that a lightpath can go through before it goes back to the electronic domain, so it corresponds to the maximum optical (without OEO conversions) hops allowed in the IP/MPLS over WSON network.

Figure 4 shows the cost of the whole network (cost), the IP/MPLS network $\left(\operatorname{cost}_{I P}\right)$ and the optical network $\left(\operatorname{cost}_{O T}\right)$, for hop constraints as a function of $r$ values and without hop constraints ('best' in the figure). It can be found that the lowest CAPEX with hop constraints (obtained for a specific $r$ value) is close to the best solution,

\begin{tabular}{|c|c|c|c|c|c|}
\hline IP/MPLS node & Class 1 & Class 2 & Class 3 & Class 4 & Class 5 \\
\hline $\begin{array}{c}\text { Switch cap. } \\
\text { (Gbps) }\end{array}$ & 160 & 320 & 640 & 1280 & 2560 \\
\hline Port no & 4 & 8 & 16 & 32 & 64 \\
\hline Cost $(\mathrm{cu})$ & 3 & 4.5 & 6.5 & 22.5 & 50.19 \\
\hline
\end{tabular}

which demonstrates the effectiveness of our heuristic algorithm. This is due to the fact that our heuristic algorithm routes demands ensuring minimum added cost (it routes new connection demands in as less lightpaths as possible to consume minimum optical resources), and More $r$-hops lightpaths establishment strategy reduces the number of new lightpaths to be established in a path, and $K$ paths can be chosen for any new lightpath.

Under hop constraints, cost ${ }_{I P}$ decreases, and cost $_{O T}$ increases in all three considered network topologies, when $r$ increases. This is because by letting lightpaths go through more optical hops, more optical bypass of OXC nodes is used, and then the electrical processing of IP/MPLS routers is reduced. This finally leads to less traffic switched in IP/MPLS routers (see figure 5a for average switched traffic in a router decreases when $r$ increases), less lightpaths established (less IP/MPLS ports used), and hence reduced IP/MPLS network CAPEX. However, longer lightpaths established for high $r$ values lead to optical network CAPEX increase. It is because when long lightpaths are used, traffic carried on it consumes more optical 


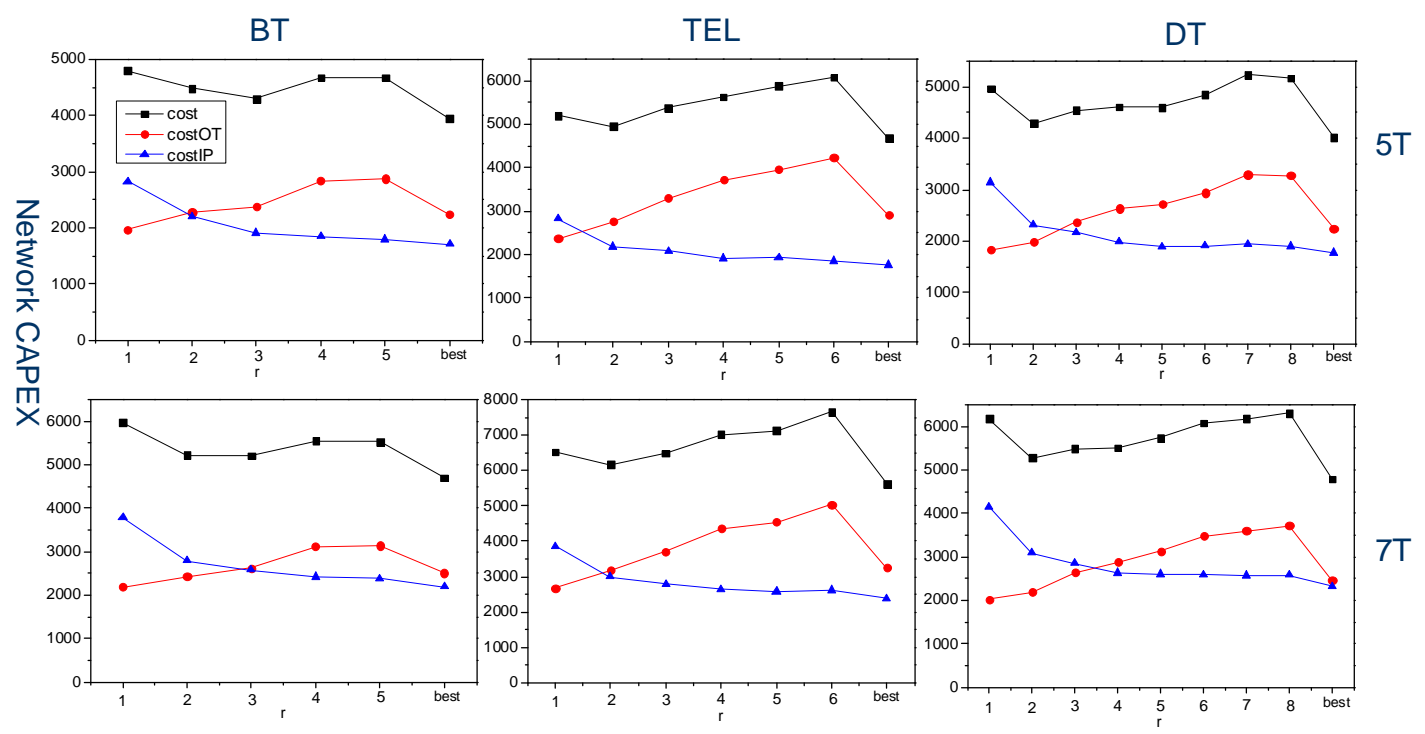

Figure 4 Cost comparisons for different $r$ and demand profiles for BT, TEL and DT networks.

BT

TEL

DT

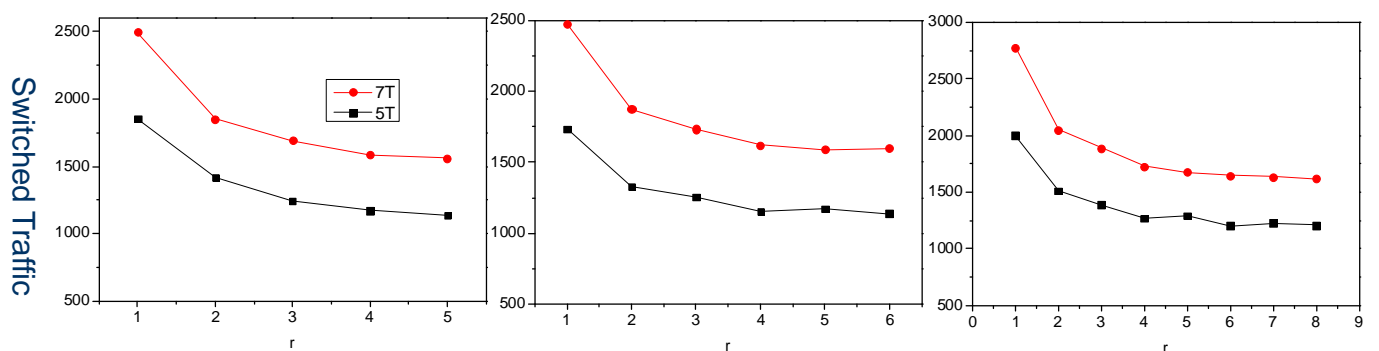

(a) Average Switched traffic in each router
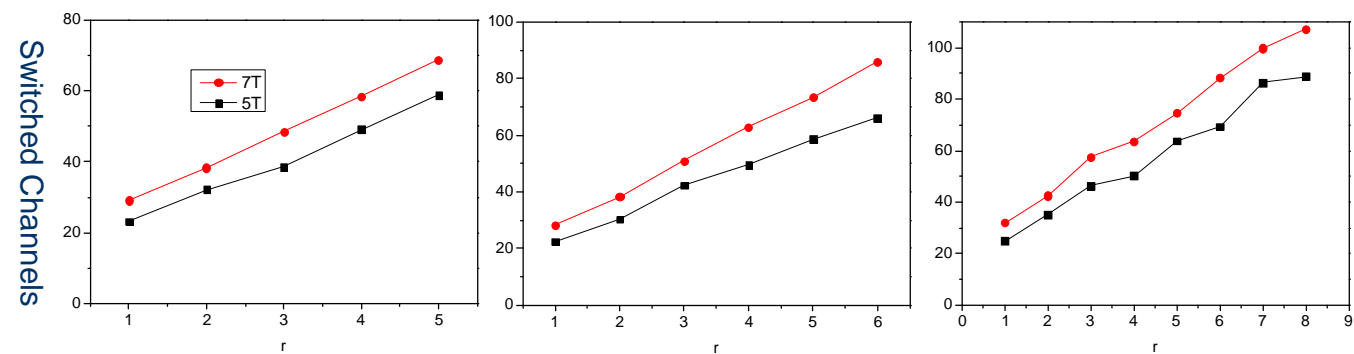

(b) Average Switched wavelength channels in each OXC

Figure 5 Average Switched wavelength channels in each OXC and Average switched traffic in each router.

resources (such as wavelength resources, see its increase with $r$ in figure $5 \mathrm{~b}$ ). In addition, long lightpaths make traffic have less chance to be groomed into IP/MPLS routers and a higher number of lightpaths is necessary. All these factors make WSON network CAPEX increase. According to their opposite CAPEX variation trends, it can be reached an optimum tradeoff between IP/MPLS network and WSON CAPEX by choosing the appropriate $r$ value. For example, in BT, TEL and DT networks with 5T demand profiles, setting $r$ to, respectively, 2,2 and 3 , is satisfactory from both, IP/MPLS and WSON, network points of view (see figure. 4). Also under hop constraints, it can be found that when $r$ increases, the whole network cost first decreases, and then rises again as a general trend. For each network and demand scenario, there is 
a specific value of $r$ that gives the minimal cost. For example, $r=3$ is the optimum value in BT network (for both 5Tbps and 7Tbps demand profiles), while $r=2$ is the right value for TEL and DT networks. This behavior is due to the fact that total network CAPEX variation is driven by CAPEX changing slopes of IP/MPLS and WSON parts when $r$ changes. For small values of $r$, the rate of $\cos t_{I P}$ reduction is higher than that of $\cos t_{O T}$ increase when $r$ becomes bigger; on the other hand, for big values of $r$, the rate of $\cos t_{I P}$ reduction is generally smaller than that of $\cos t_{O T}$ increase. In fact, the rate of $\cos t_{I P}$ reduction slows down when $r$ increases, while the slope of $\cos t_{O T}$ increase keeps almost constant for every $r$ values in our simulations.

\section{Conclusion}

In this paper, we have analyzed the design of IP/MPLS over WSON multilayer networks with hop constraints. The key point of this analysis is on limiting the maximum optical transparent reach in the network, aiming to reduce the total network CAPEX, and reach a balance between the IP/MPLS network and WSON network CAPEX. A full cost model is given, and an efficient heuristic algorithm by routing lightpaths with hop constraints has been presented. Illustrative numerical results demonstrate that there exists an appropriate $r$ value, which can get an optimum tradeoff between IP/MPLS networks and WSON CAPEX, and also leads to cost savings for the whole network CAPEX.

\section{Acknowledgement}

It was jointly supported by Jiangsu Government Scholarship for Overseas studies, China, the open fund of State Key Laboratory of Advanced Optical Communication Systems and Networks,
Shanghai Jiao Tong University, China (No. 2015GZKF03006), and Research Center of Optical Communications Engineering \& Technology, Jiangsu Province (No. ZSF0201).

\section{References}

[1]. A. Gumaste, S. Akhtar, "Evolution of PacketOptical Integration in Backbone and Metropolitan High-Speed Networks: A Standards Perspective", IEEE Comm. Mag., Vol. 51, No. 11, Pp. 105-111, 2013.

[2]. F. Rambach, B. Konrad, L. Dembeck, U. Gebhard, M. Gunkel, M. Quagliotti, L. Serra, and V. LópezA, "Multilayer Cost Model for Metro/Core Networks", J. OPT. COMMUN. NETW., Vol. 5, No. 3, Pp.210-225, 2013.

[3]. R. Duque, V. López, A. González, O. González, J.P. Fernández-Palacios, "Cost evaluation of the integration of IP/MPLS and WDM elements", OFC/NFOEC 2013, NM3F.3.pdf

[4]. C. Lee, J.K.K. Rhee, "Traffic Grooming for IP-Over-WDM Networks: Energy and Delay Perspectives", J. OPT. COMMUN. NETW. Vol. 6, No. 2, Pp.96-103, 2014.

[5]. K. Zhu, B. Mukherjee, "Traffic Grooming in an Optical WDM Mesh Network", IEEE Journal on Selected Areas in Communications, Vol. 20, No. 1, Pp. 122-133, 2002.

[6]. F. Agraz, L. Velasco, J. Perelló, M. Ruiz, S. Spadaro, G. Junyent, J. Comellas, "Design and implementation of a GMPLS-controlled grooming-capable optical transport network", IEEE/OSA Journal of Optical Communications and Networking, Vol. 1, No. 2, Pp. 258-269, 2009.

[7]. J. Comellas, R. Martínez, J. Prat, V. Sales, G. Junyent, "Integrated IP/WDM Routing in GMPLS-Based Optical Networks", IEEE Network, Vol. 17, No. 2, Pp. 22-27, 2003.

[8]. M. Jinno, H. Takara, B. Kozicki, Y. Tsukishima, Y. Sone, S. Matsuoka, "Spectrum-efficient and scalable elastic optical path network: architecture, benefits, and enabling technologies", IEEE Communications Magazine, Vol.47, No.11, Pp. 66-73, 2009.

[9]. Y.R. Huang, J. P. Heritage, B. Mukherjee, "Connection Provisioning With Transmission Impairment Consideration in Optical WDM Networks With High-Speed Channels", IEEE Journal of Lightwave Technology, Vol. 23, No. 3, Pp.982-993, 2005

[10]. F.Q. Liu, H.L. Feng, Y.Q. Qian, "Impairment-aware traffic grooming in WDM optical networks", Chin. Opt. Lett. Vol. 8, No. 5, Pp. 460-463, 2010

[11].T. Zami, "Physical impairment aware planning of next generation WDM backbone networks", 
OFC/NFOEC 2012, OW3A.3.pdf, 2012.

[12].J. Zhao, S. Subramaniam, M. BrandtPearce, "Intradomain and interdomain QoT-aware RWA for translucent optical networks", IEEE/OSA Journal of Optical Communications and Networking, vol. 6, no. 6 , pp. 536-548, 2014

\section{Biographies:}

Fengqing Liu, received his B.E. and M.S. degrees in computer science from Harbin Engineering University, China, in 1997 and 2000 respectively. He received his PH.D. in Electronic Engineering from Shanghai Jiaotong University, China, in 2004. His research interests are in the area of IP over optical networking, especially logical topology design, traffic grooming, and performance analysis. $\mathrm{He}$ is an associate professor of Nanjing University of Posts and Telecommunications.

Jaume Comellas, received the M.S. (1993) and Ph.D. (1999) degrees in Telecommunications Engineering from Universitat Politècnica de Catalunya (UPC), Spain. His current research interests focus on IP over optical networking topics. He has participated in many research projects funded by the Spanish government and the European Commission. He has co-authored more than 120 research articles in international journals and conferences. He is an associate professor at the Signal Theory and Communications Department of UPC. 